\title{
Glucocorticoid use and complications following immune checkpoint inhibitor use in melanoma $\leqslant$
}

\author{
Authors: Kapil Agarwal, ${ }^{A}$ Nadia Yousaf ${ }^{B}$ and Daniel Morganstein ${ }^{C}$
}

\section{Background}

Immune checkpoint inhibitors have demonstrated benefit in the treatment of cancer, but are associated with toxicities, which often require treatment with glucocorticoids.

\section{Aims}

We aimed to determine the prevalence of glucocorticoid use in patients treated with immune checkpoint inhibitors for melanoma in a single centre.

\section{Methods}

We performed a retrospective review of patients with advanced melanoma treated with an immune checkpoint inhibitor between September 2010 and January 2017. Patients treated with glucocorticoids had a cumulative dose and duration of glucocorticoid treatment calculated. New onset hyperglycaemia was also identified.

Results

Of 412 patients receiving immune checkpoint therapy, 157 (38\%) required glucocorticoids to treat toxicities. The median cumulative glucocorticoid dose was $2,795 \mathrm{mg}$ (prednisolone equivalent) with a median duration of 61 days. Twenty-seven patients receiving glucocorticoids were noted to develop new onset hyperglycaemia.

\section{Conclusions}

Immune-related adverse events frequently occur in patients treated with immune checkpoint inhibitors. Consequently, patients receive prolonged courses of glucocorticoids. Awareness of glucocorticoid-induced side effects is required.

KEYWORDS: Checkpoint inhibitor, glucocorticoids, melanoma, hyperglycaemia, adverse events

DOI: $10.7861 /$ clinmed.2018-0440

\section{Introduction}

Immunotherapy

Immunotherapy with immune checkpoint inhibitors (ICPI) has led to a paradigm shift in the management of a number

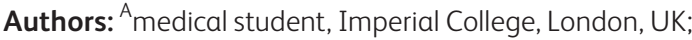
${ }^{B}$ consultant medical oncologist, Royal Marsden Hospital, London, UK; ' Consultant endocrinologist, Chelsea and Westminster Hospital, London, UK of malignancies, including melanoma, renal cell carcinoma and non-small cell lung cancer (NSCLC). ${ }^{1,2}$ ICPIs promote T-cell activation and can lead to an improved overall survival compared to chemotherapy. ${ }^{1,2}$ Monoclonal antibodies blocking both the cytotoxic T-lymphocyte-associated protein 4 (CTLA-4) and programmed cell death-1 (PD-1) / programmed cell death ligand-1 (PD-L1) pathways are now approved for the treatment of several cancers, and have emerged as first line therapy for metastatic melanoma, as well as having benefit in the adjuvant setting. ${ }^{3,4}$ However, the alterations of the immune system through immunotherapy can result in toxicity, termed immune-related adverse events (IRAEs). ${ }^{5,6}$ ICPI-induced IRAEs affect many organ systems, however, some of the most commonly affected organs include the gastrointestinal tract, skin, liver and endocrine glands. ${ }^{5,7}$ Consequently, common side effects of ICPI treatment include colitis, rash, hepatitis and endocrinopathies. ${ }^{7-9}$

Among those with melanoma, combination therapy with ipilimumab and nivolumab resulted in the greatest overall survival rate as compared to either agent alone, however, over half of the patients (59\%) developed grade 3 or 4 adverse events. ${ }^{10}$ In contrast, treatment with a single ICPI resulted in a lower proportion of patients developing IRAEs. ${ }^{10}$ Comparisons of anti-PD-1 antibody treatment against anti-CTLA-4 antibody treatment also indicate the anti-PD-1 antibodies have a lower percentage of grade 3 or 4 adverse events ( $22 \%$ in patients treated with nivolumab compared to $28 \%$ in patients treated with ipilimumab), while there are also differences in the pattern of adverse events between the CTLA- 4 and PD-1 inhibitors. ${ }^{10,11}$ With the exception of endocrinopathies, most IRAEs are reversible with early detection and appropriate treatment. ${ }^{7}$ Although the specific management for each toxicity varies, in general there is consistency in treating the IRAEs depending on the severity. Grade 1 IRAEs are typically managed with supportive measures and close monitoring while continuing ICPI therapy. ${ }^{7,12}$ Grade 2 IRAEs are usually managed by withholding ICPI therapy and initiating oral steroids with the dose tapered down over subsequent weeks. ${ }^{7,12}$ In grade 3 and 4 IRAEs, ICPI therapy should be suspended with permanent discontinuation considered, while high-dose corticosteroids should also be commenced, with the dose tapered down over subsequent weeks. ${ }^{7,12}$ In the case of endocrinopathies, ICPI therapy can frequently be continued but appropriate hormone replacement therapy should be commenced, although occasionally high doses of corticosteroids are also required, for example in the case of significant pituitary enlargement. ${ }^{12}$ 
While corticosteroids are generally the first-line treatment for reversing inflammation due to immunotherapy, increasingly other disease-modifying drugs are used, such as infliximab or mycopheleate mofetil. ${ }^{13}$ Despite this, high doses of corticosteroids are often required, which exposes patients to the associated risks of glucocorticoid-induced diabetes and osteoporosis. ${ }^{14,15}$

This study therefore audited a cohort of patients treated with ICPIs for melanoma to determine the prevalence of glucocorticoid use, the dose and duration of therapy used and development of glucocorticoid-induced hyperglycaemia.

\section{Methods}

\section{Study population}

In this retrospective audit, patients treated with one or more ICPIs for advanced melanoma at the Royal Marsden Hospital (RMH) between September 2010 and January 2017 were included for analysis. All data pertaining to ICPI treatment and corticosteroid treatment was collected until April 2018, to account for patients with ongoing treatment. Approval for this study was obtained from the Royal Marsden Clinical Audit Committee.

\section{Data collection}

Through analysis of the medical records on the RMH electronic patient record (EPR), all 412 patients were assessed for treatment with glucocorticoids. Relevant data was extracted and recorded in an audit tool, in the form of an MS Excel spreadsheet.

All patients prescribed oral or intravenous glucocorticoids were identified and the indications for glucocorticoid administration were determined by analysing their medical case notes. For patients where glucocorticoids were administered for melanoma progression such as cerebral metastases or spinal metastases, this was noted but the cumulative corticosteroid exposure was not calculated. However, for patients where glucocorticoids were administered to treat an IRAE, this was recorded and the cumulative corticosteroid exposure was also calculated.

Due to limitations with the case documentation available regarding corticosteroid doses and adjustments to treatment, the following assumptions were made when collecting the data.

> On occasions where a patient was admitted to hospital for intravenous corticosteroid treatment with methylprednisolone and the dose administered was not recorded, it was assumed that a dose of $1 \mathrm{mg} / \mathrm{kg}$ was given in accordance with guidelines. $^{12,13}$

> Where the glucocorticoid dose was tapered down but exact dates and doses were not documented, it was assumed the dose was tapered down at even time intervals by $5 \mathrm{mg}$ per interval, in line with standard clinical practice.

$>$ Where patients received corticosteroid treatment, it was assumed this was for IRAEs unless explicitly mentioned that the corticosteroids were for other indications.

As several different corticosteroids were used to treat IRAEs across patients, all corticosteroid doses were converted into a prednisolone equivalent, based on the relative potencies and pharmacodynamics of each corticosteroid. ${ }^{16,17}$ Where budesonide was prescribed, this corticosteroid dose was excluded due to the low systemic absorption of budesonide, as although there may be sufficient absorption to result in adrenal suppression, this is difficult to quantify. ${ }^{18,19}$ The total number of days of corticosteroid treatment and the total amount of corticosteroid as prednisolone (or equivalent) was calculated, giving a cumulative corticosteroid exposure. Due to prolonged glucocorticoid treatment, some patients developed adrenal suppression and subsequently required physiological replacement doses of glucocorticoids or developed adrenal insufficiency due to an endocrinopathy. Therefore, glucocorticoid doses of $5 \mathrm{mg}$ or less (prednisolone equivalent) administered for replacement were not included when calculating cumulative corticosteroid exposure. If a patient received more than one course of corticosteroids then all courses were included to calculate the duration and cumulative dose, in order to give an accurate assessment of total exposure. Use of topical glucocorticoids were not included in this analysis.

Laboratory records were also checked to determine new onset hyperglycaemia; defined as a random glucose $>11.1 \mathrm{mmol} / \mathrm{L}$ in patients without pre-existing diabetes. Random laboratory venous glucose readings routinely obtained at outpatient visits and prior to the administration of each cycle of immunotherapy were utilised to detect hyperglycaemia, as this was representative of clinical practice.

\section{Statistical analysis}

To assess distribution of the corticosteroid dose and duration, the D'Agostino-Pearson normality test was performed, with median values obtained. To compare duration and dose of corticosteroid treatment between patients who developed newonset hyperglycaemia with patients who remained euglycaemic, a Mann-Whitney $U$ test was performed $(p<0.05)$. To compare baseline glucose values between patients who developed new onset hyperglycaemia and patients who remained euglycaemic, an unpaired t-test was performed $(p<0.05)$. All statistical analyses were performed using GraphPad Prism Software version 5.0 (GraphPad Software, San Diego, USA). For descriptive statistics, functions such as 'COUNTIF' and 'SUM' within MS Excel 2016 (Microsoft, Redmond, USA) were used.

\section{Results}

\section{Prevalence of corticosteroid use}

Four-hundred and twelve patients received treatment with ICPIs for metastatic melanoma. The median age was 59 years, with 229 males (56\%) and 183 females (44\%). Two-hundred and eighty-six patients (69\%) received anti-CTLA-4 antibody treatment, 69 patients (17\%) received anti-PD-1 antibody treatment and 57 patients $(14 \%)$ received treatment either sequentially or in combination with both anti-CTLA-4 and anti-PD-1 antibodies. Of the initial 412 patients, 157 patients (38\%) received oral or intravenous corticosteroids greater than $5 \mathrm{mg}$ prednisolone equivalent directly for IRAEs, while 103 patients (25\%) received corticosteroids for oncological or other purposes, most frequently due to cerebral metastases. The remaining 152 patients ( $37 \%$ ) were documented not to have received oral or intravenous corticosteroids for any purpose (Fig 1).

Of the 157 patients, two were treated entirely outside our institution so no details of dose were available. Among the remaining 155 patients receiving corticosteroids for IRAEs, the median duration was 61 days, with a maximum duration of 974 days and a minimum duration of 3 days (Fig 2a). The median 


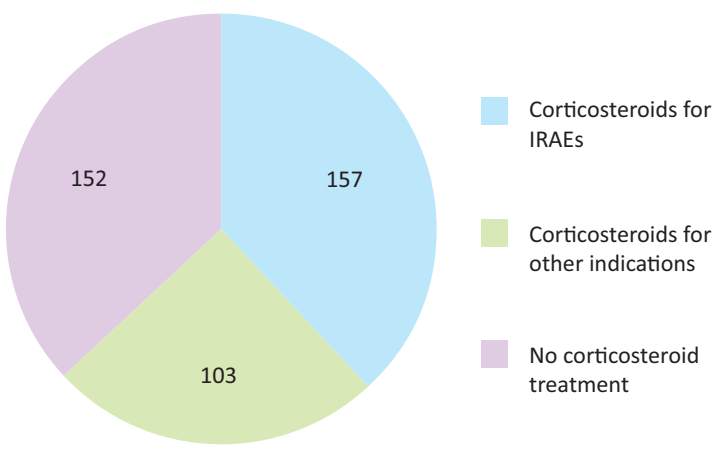

Fig 1. Number of patients treated with corticosteroids $(n=412)$. IRAEs $=$ immune-related adverse events.

cumulative corticosteroid dose was 2,795 mg prednisolone equivalent, with a maximum cumulative dose of $24,254 \mathrm{mg}$ and a minimum cumulative dose of $25 \mathrm{mg}$ (Fig 2b). Prednisolone was the most commonly used steroid, although methylprednisolone, dexamethasone and hydrocortisone were also used (Fig 2c).

Fifty-six patients received more than one course of corticosteroid, defined as a course that was tapered down and stopped, then recommenced.

\section{Glucose monitoring and new onset hyperglycaemia}

Of the 157 patients receiving corticosteroids for IRAEs, 144 $(92 \%)$ patients had a random blood glucose measurement at the initiation of corticosteroid treatment or within the month prior to starting corticosteroids. The majority of these patients had further glucose monitoring during their treatment, mostly in the outpatient setting although some patients also had glucose monitoring during admissions to hospital as inpatients. Four patients had no further glucose monitoring and for a further three patients, assessment of glucose monitoring could not be made due to transfer of care to other hospitals. After excluding seven patients with pre-existing diabetes and those for whom data regarding further glucose monitoring was not available, 130 patients' records were analysed for new onset hyperglycaemia. Just over one-fifth (27) of these 130 patients were noted to develop new onset hyperglycaemia, while the remaining 103 patients had no measured glucose levels greater than $11.1 \mathrm{mmol} / \mathrm{L}$.

\section{Factors predicting hyperglycaemia}

For patients receiving corticosteroids for IRAEs, a significant difference was found between the average pre-treatment glucose value in patients who developed glucocorticoidinduced hyperglycaemia compared to those who did not. The mean pre-treatment glucose value in patients who developed hyperglycaemia was $6.7 \mathrm{mmol} / \mathrm{L}$ in comparison to the mean pre-treatment glucose value of $5.8 \mathrm{mmol} / \mathrm{L}$ for patients who did not develop subsequent hyperglycaemia ( $p=0.001$; Fig $3 a$ ).

A significant difference was also found in the median dose and duration of corticosteroid treatment between patients who developed hyperglycaemia and those who did not. The median cumulative corticosteroid dose (as prednisolone equivalent) in patients who developed hyperglycaemia was 5,631 mg compared to a median cumulative corticosteroid dose of $2,200 \mathrm{mg}$ in
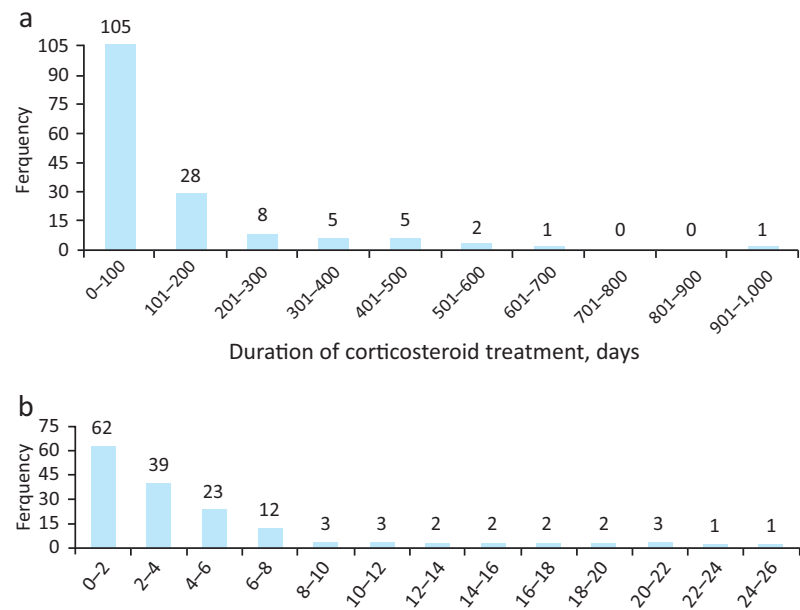

Cumulative dose of corticosteroid received, $x 1,000 \mathrm{mg}$ as prednisolone equivalent

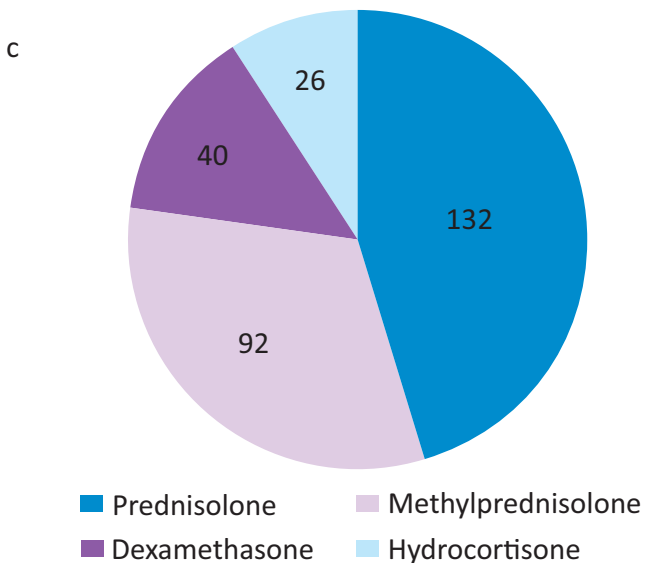

Fig 2. Corticosteroid use. a) Duration of corticosteroid treatment for patients receiving glucocorticoids due to immune-related adverse events. b) Cumulative dose of corticosteroid received by patients being treated for immune-related adverse events. c) Number of patients receiving each type of corticosteroid. Many patients received more than one different type of corticosteroid and hence the total number exceeds the number of patients receiving corticosteroids.

patients who did not develop hyperglycaemia $(p<0.0001$; Fig $3 b$ ). The median duration of corticosteroid treatment in patients who developed hyperglycaemia was 124 days compared to a median duration of 52 days in patients who did not develop hyperglycaemia ( $p<0.0001$; Fig 3c).

\section{Discussion}

\section{Prevalence of glucocorticoid use}

In this audit, $38 \%$ of patients received oral or intravenous corticosteroids greater than $5 \mathrm{mg}$ prednisolone equivalent directly for IRAEs. This is similar to a retrospective analysis conducted at the Memorial Sloan Kettering Cancer Centre, where 35\% of patients treated with ipilimumab for melanoma received systemic corticosteroids for IRAEs. ${ }^{20}$ In a systematic review, Garant et al evaluated observational studies where patients were administered 

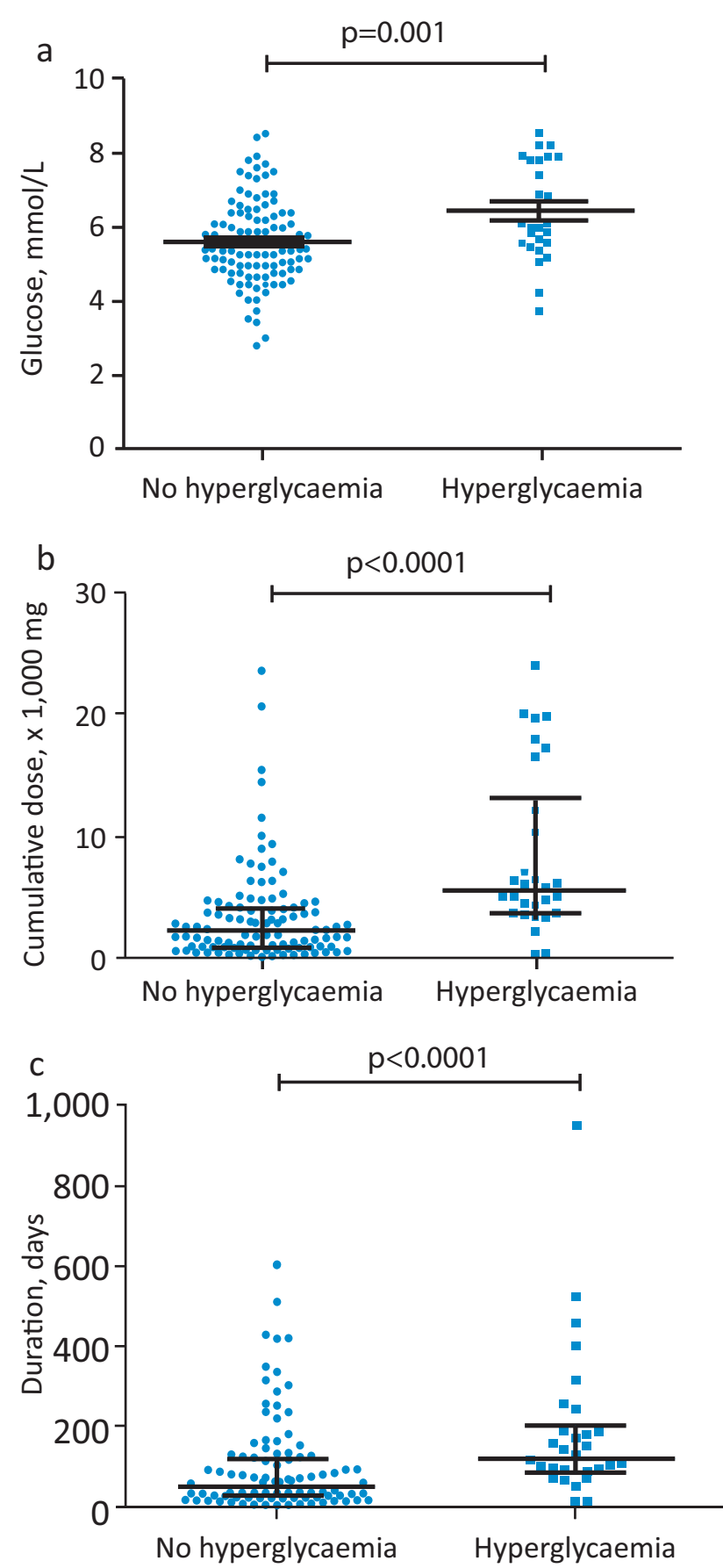

Fig 3. Comparisons of patients that developed glucocorticoid-induced hyperglycaemia and those who did not. a) Comparison of the pretreatment random glucose values; mean \pm standard error of mean. b) Comparison of cumulative corticosteroid dose (as prednisolone equivalent); median and interquartile range (IQR). c) Comparison of duration of glucocorticoid treatment; median and IQR.

corticosteroids for IRAEs following ICPI therapy. ${ }^{21}$ While the proportion of patients receiving corticosteroids for IRAEs varied slightly between the studies, corticosteroids were generally prescribed for around one-third of patients, comparable to the results observed in this audit. ${ }^{21,22}$
The cumulative dose and duration of corticosteroid administration for IRAEs was often much greater than more typical clinical indications for corticosteroid use. Patients receiving corticosteroids for common medical conditions such as chronic obstructive pulmonary disease and rheumatoid arthritis typically require $2-4$ weeks of corticosteroid treatment, with a cumulative corticosteroid dose well below $500 \mathrm{mg}$ prednisolone equivalent. In such instances, the guidelines do not necessitate monitoring for glucocorticoid-induced complications due to the short-term, low-dose corticosteroid treatment. However, in a condition such as temporal arteritis, for example, patients can receive corticosteroids for up to 1 year or more, with the cumulative corticosteroid dose potentially reaching in excess of $10,000 \mathrm{mg}$ prednisolone equivalent. National guidelines recommend risk assessments and appropriate monitoring for side effects of steroid use, especially hyperglycaemia and osteoporosis, particularly with more prolonged courses, and this data highlights the importance of such screening in patients requiring glucocorticoids for management of the toxicities of checkpoint inhibitor therapy in cancer.

\section{Hyperglycaemia}

The results of this study demonstrated a high rate of new onset hyperglycaemia occurring in response to corticosteroid treatment. The results of this study also showed that patients who developed hyperglycaemia had a higher mean glucose value at corticosteroid initiation compared to those who did not develop hyperglycaemia. However, other markers of pre-existing hyperglycaemia such as glycated haemoglobin were not routinely monitored in this cohort. Patients who developed hyperglycaemia also received a higher median cumulative dose and duration of corticosteroid compared to those who did not develop hyperglycaemia. Despite a statistically significant difference in the pre-treatment glucose values between patients who developed new onset hyperglycaemia and patients who did not, it is unlikely that this finding will be of significant clinical utility, given the large range of baseline glucose values and the overlapping of values between the new onset hyperglycaemia group and the normoglycaemia group. However, it does highlight the importance of assessing pre-treatment glucose metabolism to identify those at the highest risk of hyperglycaemia. Similarly, while a statistically significant difference was found in both the median cumulative corticosteroid dose and median corticosteroid duration between patients who developed hyperglycaemia and patients who did not, highlighting the need to focus screening on those needing the highest doses, (although this may not be known at the start of treatment), consideration needs to be given to screening all patients receiving glucocorticoids for the development of hyperglycaemia, pending better predictive biomarkers. The prompt detection of hyperglycaemia is even more important given that it is now emerging that a presumed immune-mediated form of diabetes, resembling type 1 diabetes can rarely occur following inhibition of the PD-1 pathway. ${ }^{23,24}$ In this study we did not collect detailed data about outcomes in the patients with hyperglycaemia, so cannot rule out the possibility that hyperglycaemia in some patients may have been a direct result of the checkpoint inhibitor. However, as we only screened those patients receiving corticosteroids, they would likely have contributed to the degree of hyperglycaemia even if any patients had developed diabetes as an immune-related adverse event. 
The study has a number of strengths. Firstly, this study audited a large sample size of patients. Secondly, the results of this audit were based upon data covering 7 years, meaning the findings are not affected by any short-term variations in either clinical practice or in the patient cohort that may have arisen. Thirdly, there was consistency in the method of data collection as all data was collected by one author.

However, there are two main limitations to this audit. Firstly, this audit only reviewed the practice at a single centre and more specifically, a single team looking after melanoma patients. However, the proportion of patients receiving glucocorticoids was similar to two other studies, suggesting the results are likely to be generalisable. Secondly, many of the results obtained in this audit are likely to be underestimates of the true values. We have not analysed use of topical glucocorticoids or those, such as budesonide, with reduced systemic absorption, while use of glucocorticoids initiated locally prior to transfer to the specialist centre have also not been included. Likewise, we have not assessed use of topical glucocorticoids, although these can be used, for example, in mild skin reactions, and can at times be associated with systemic effects. Thus, the cumulative glucocorticoid dose and duration are likely to be under-represented. However, as the reported doses are large, this further strengthens the need for careful management of side effects of glucocorticoid exposure. In addition, as the diagnosis of hyperglycaemia was made on the basis of elevated random blood glucose levels obtained during routine clinic visits, it is possible that the frequency of hyperglycaemia is an underestimate. A more targeted approach, particularly using self blood glucose monitoring may have shown the true incidence to be even higher, although we cannot rule out the possibility of stress hyperglycaemia due to acute illness as a contributor in some patients

\section{Conclusion}

The use of ICPIs for the treatment of cancer is growing. Until recently, the use of corticosteroids in oncology was mainly for short durations or in the palliative care setting. However, patterns of corticosteroid use are changing due to the widespread use of ICPIs, and a proportion of patients obtaining a durable clinical benefit. Nevertheless, this does mean that the third of patients who will require corticosteroids for IRAEs are at increased risk of developing glucocorticoid-induced complications such as hyperglycaemia and osteoporosis. In the absence of prospective studies and biomarkers to determine the optimal toxicity management strategy, current expert consensus guidelines stipulate a stepwise approach starting with high dose corticosteroids in the first instance and escalating to additional immunosuppression if needed. Future clinical trials which incorporate targeting selective inflammatory cytokines to 'switch off' toxicity are desperately needed. However, in the interim, it is important for all those involved in treating patients with immune checkpoint inhibitors, but especially oncology teams, to introduce robust pathways to screen patients requiring corticosteroids for hyperglycaemia and osteoporosis and follow national guidelines for the management of these complications.

\section{Acknowledgments}

The authors would like to acknowledge Dr Lavinia Spain who maintained the database from which patients were identified.

\section{References}

1 Drake CG, Lipson EJ, Brahmer JR. Breathing new life into immunotherapy: review of melanoma, lung and kidney cancer. Nat Rev Clin Oncol 2014;11:24-37.

2 Farkona S, Diamandis EP, Blasutig IM. Cancer immunotherapy: the beginning of the end of cancer? BMC Med 2016;14:73.

3 Force J, Salama AK. First-line treatment of metastatic melanoma: role of nivolumab. Immunotargets Ther 2017;6:1-10.

4 Guldbrandsen KF, Hendel HW, Langer SW, Fischer BM. Nuclear molecular imaging strategies in immune checkpoint inhibitor therapy. Diagnostics (Basel) 2017;7:23.

5 Michot JM, Bigenwald C, Champiat S et al. Immune-related adverse events with immune checkpoint blockade: a comprehensive review. Eur J Cancer 2016;54:139-48.

6 Puzanov I, Diab A, Abdallah K et al. Managing toxicities associated with immune checkpoint inhibitors: consensus recommendations from the Society for Immunotherapy of Cancer (SITC) Toxicity Management Working Group. J Immunother Cancer 2017;5:95.

7 Spain L, Diem S, Larkin J. Management of toxicities of immune checkpoint inhibitors. Cancer Treat Rev 2016;44:51-60.

8 Villadolid J, Amin A. Immune checkpoint inhibitors in clinical practice: update on management of immune-related toxicities. Transl Lung Cancer Res 2015;4:560-75.

9 Kumar V, Chaudhary N, Garg M et al. Current diagnosis and management of immune related adverse events (irAEs) induced by immune checkpoint inhibitor therapy. Front Pharmacol 2017;8:49.

10 Hodi FS, Chiarion-Sileni V, Gonzalez R et al. Nivolumab plus ipilimumab or nivolumab alone versus ipilimumab alone in advanced melanoma (Checkmate 067): 4-year outcomes of a multicentre, randomised, phase 3 trial. Lancet Oncol 2018;19:1480-92.

11 Barroso-Sousa R, Barry WT, Garrido-Castro AC et al. Incidence of endocrine dysfunction following the use of different immune checkpoint inhibitor regimens: a systematic review and metaanalysis. JAMA Oncol 2018;4:173-82.

12 Brahmer JR, Lacchetti C, Schneider B] et al. Management of immune-related adverse events in patients treated with immune checkpoint inhibitor therapy: American Society of Clinical Oncology Clinical Practice Guideline. J Clin Oncol 2018;36:1714-68.

13 Haanen JBAG, Carbonnel F, Robert $C$ et al. Management of toxicities from immunotherapy: ESMO Clinical Practice Guidelines for diagnosis, treatment and follow-up. Ann Oncol 2017;28 (suppl 4):iv119-42.

14 Oray M, Abu Samra K, Ebrahimiadib N, Meese H, Foster CS. Long-term side effects of glucocorticoids. Expert Opin Drug Saf 2016;15:457-65.

15 Klein GL. The effect of glucocorticoids on bone and muscle. Osteoporos Sarcopenia. 2015;1:39-45.

16 Zoorob RJ, Cender D. A different look at corticosteroids. Am Fam Physician. 1998;58:443-50.

17 Bonaventura A, Montecucco F. Steroid-induced hyperglycemia: An underdiagnosed problem or clinical inertia? A narrative review. Diabetes Res Clin Pract 2018;139:203-20.

18 Iborra M, Alvarez-Sotomayor D, Nos P. Long-term safety and efficacy of budesonide in the treatment of ulcerative colitis. Clin Exp Gastroenterol 2014;7:39-46.

19 Campieri M, Ferguson A, Doe W, Persson T, Nilsson LG. Oral budesonide is as effective as oral prednisolone in active Crohn's disease. The Global Budesonide Study Group. Gut 1997:41:209-14.

20 Horvat TZ, Adel NG, Dang T et al. Immune-related adverse events, need for systemic immunosuppression, and effects on survival and time to treatment failure in patients with melanoma treated with ipilimumab at Memorial Sloan Kettering Cancer Center. J Clin Oncol 2015;33:3193-8. 
21 Garant A, Guilbault C, Ekmekjian T et al. Concomitant use of corticosteroids and immune checkpoint inhibitors in patients with hematologic or solid neoplasms: A systematic review. Crit Rev Oncol Hematol 2017;120:86-92.

22 Williams KJ, Grauer DW, Henry DW, Rockey ML. Corticosteroids for the management of immune-related adverse events in patients receiving checkpoint inhibitors. J Oncol Pharm Pract 2019;25: 544-50.

23 Marchand L, Thivolet A, Dalle $S$ et al. Diabetes mellitus induced by PD-1 and PD-L1 inhibitors: description of pancreatic endocrine and exocrine phenotype. Acta Diabetol 2019;56:441-8.
24 Clotman K, Janssens K, Specenier P, Weets I, De Block CEM Programmed cell death-1 inhibitor-induced type 1 diabetes mellitus. J Clin Endocrinol Metab 2018;103:3144-54.

Address for correspondence: Dr Daniel Morganstein, Chelsea and Westminster Hospital, 369 Fulham Road, London SW10 9NH, UK.

Email: d.morganstein@ic.ac.uk

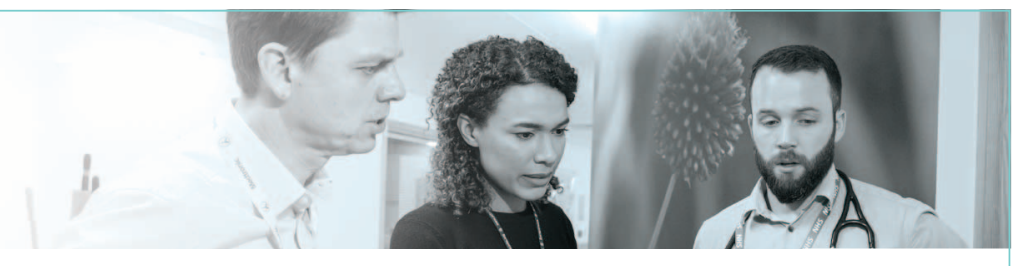

Medical Care

\section{Are you involved in planning medical services?}

Medical Care is the online evolution of the well-known RCP publication Consultant physicians working with patients. Written by leading medical specialty experts, it is a comprehensive web-based resource for the efficient and effective design of medical services.
Medical Care has been designed to help those involved in the planning and provision of medical services to get a clearer picture of the specialty services that need to be in place to provide great patient care.

The resource looks in detail at the services delivered by 29 medical specialties.

It also includes overarching themes covering the development of physicians and their teams.

\section{www.rcpmedicalcare.org.uk}

